\title{
Características de las investigaciones presentadas en el Congreso Peruano de Educación Médica
}

\author{
Guido Bendezú-Quispe, Sarita Quispe-Colquepisco, Tania Acevedo-Villar, Fanny Bastidas-García
}

La educación médica debe optimizar las capacidades de todos los estudiantes y profesionales desarrollando la motivación y la orientación para el aprendizaje futuro, proporcionando bases para el desarrollo profesional y brindando a la sociedad profesionales competentes acordes a las exigencias y necesidades de su realidad $[1,2]$. En este sentido, la Asociación Peruana de Facultades de Medicina (ASPEFAM) es el organismo asesor y coordinador en el área de la enseñanza médica en el país, promueve y coordina el desarrollo de las instituciones que la integran en función de las necesidades de salud del país. Entre sus funciones recae la acreditación de las facultades de medicina y la realización del examen nacional de medicina. Realiza diversos talleres, simposios y, desde 2005, el Congreso Peruano de Educación Médica, espacio de análisis y discusión sobre la situación actual, los desafíos y las perspectivas de la formación médica nacional e internacional [3].

Con el objetivo de conocer las características de las investigaciones presentadas en el Congreso Peruano de Educación Médica durante los años 2007 y 2010, se generó una base de datos con el total de resúmenes presentados a dicho evento, registrándose el número de autores, título y filiación institucional. Los trabajos se agruparon según las categorías establecidas en los congresos de la Sociedad Española de Educación Médica [4]. Asimismo se realizó una búsqueda en Google Scholar de los resúmenes que posteriormente se publicaron en revistas médicas, mediante una estrategia de combinación de términos de búsqueda y apellidos de los autores, previamente empleada para la búsqueda de publicaciones duplicadas [5].

Se presentaron un total de 142 trabajos de investigación. La media de autores por trabajo fue de 3,7 \pm 2 ,4 (rango: $1-13$ autores). La filiación institucional más frecuente fue la Universidad Nacional Mayor de San Marcos (23,2\%), seguida de la Universidad Peruana de Ciencias Aplicadas (14,1\%). Los trabajos sobre formación de grado $(41,6 \%)$ fueron los más habituales, seguidos por los de metodología docente $(26,1 \%)$ y desarrollo curricular $(12,7 \%)$. Sólo cuatro trabajos de investigación $(2,8 \%)$ se publicaron en una revista científica, en todos los casos de difusión nacional.

Se evidencia una participación importante en el número de investigaciones, pero una baja frecuencia de publicación de los trabajos presentados en el Congreso Peruano de Educación Médica, lo que determina la no visibilidad de la investigación hecha en el país sobre esta materia.

Consideramos que las revistas médicas peruanas serían un buen espacio para difundir las investigaciones realizadas en el área de la educación médica. Asimismo, la realización de congresos de educación médica en Perú son ámbitos importantes para el intercambio de opiniones y experiencias de mejora de la educación médica en el país. Una mayor difusión de la importancia de la publicación científica en los participantes a estos eventos y el desarrollo de una revista peruana sobre educación médica podrían favorecer una mayor difusión de las investigaciones.

Bibliografía

1. Boelen C. Medical education reform: the need for global action. Acad Med 1992; 67: 745-9.

2. Epstein RM. Assessment in medical education. N Engl J Med 2007; 356: 387-96.

3. Asociación Peruana de Facultades de Medicina (ASPEFAM). Reseña. URL: http://www.aspefam.org.pe/historia.htm. [03.06.2014]

4. Sociedad Española de Educación Médica (SEDEM). Congresos SEDEM. URL: http://www.sedem.org/styled. [10.06.2014].

5. Mayta-Tristán P, Mezones-Holguín E. Aclaración editorial Rev Peru Med Exp Salud Publica 2009; 26: 411-2.
Sociedad Científica Médico Estudiantil Peruana, SOCIMEP (G. Bendezú-Quispe). Sociedad Científica de Estudiantes de Medicina de ICa, SOCEMI; Universidad Nacional San Luis Gonzaga; Ica (G. Bendezú-Quispe, S. QuispeColquepisco, T. Acevedo-Villar). Sociedad Científica de Estudiantes de Medicina de la Universidad de San Martín de Porres; Universidad de San Martín de Porres (F. BastidasGarcía). Lima, Perú.

Correspondencia: Guido Bendezú Quispe. Avda. Salaverry, 1230. Dpto. 402 B. Jesús María, Lima, Perú.

E-mail:

guidobq@gmail.com

(c) 2015 FEM 